\title{
Airway circulation in health and disease
}

\author{
A. Lockhart*, R. Marthan**, N. Charan+, P. Paré ${ }^{++}$
}

The third biennial meeting of the da Vinci Society for the Study of the Airway Circulation was held at the University of Bordeaux, France, from September 12-14, 1995. There were 44 participants from nine different countries. The topic of the meeting was "Airway Circulation in Health and Disease: Interaction between Airway Vasculature and Pulmonary Smooth Muscles". Thirty one presentations were given and these were followed by lively discussions. Guest speaker, Paul Vanhoutte, gave a stimulating talk entitled "Epithelium, Endothelium and Smooth Muscles in Airway Walls". Old topics that had been at the forefront some decades ago were revisited and new insights into the physiology and pathophysiology of the airway circulation were discussed.

\section{Haemodynamics of the airway circulation}

The site of anastomoses between the bronchial and the pulmonary circulation is still the subject of debate. Striking angiogenesis of the airway circulation and medial hypertrophy of small pulmonary arteries have been observed 4 months after ligation of the left pulmonary artery in dogs. Using the sudden occlusion technique to measure pre- and postcapillary pulmonary vascular resistance in the in situ perfused left lower lobe of dogs, R. Michel was able to localize anastomoses between the bronchial and the pulmonary circulations at the precapillary level. There may be similarities between these findings and those from a study performed by D. Schraufnagel in rats. The rats, made cirrhotic by ligation of the bile duct, developed a striking increase of the vasa vasorum of the pulmonary arteries, as demonstrated by use of plastic casting. P. Paré has also investigated the extent of these anastomoses in open-chested, anaesthetized sheep. Radiolabelled red blood cells were injected into the common bronchial artery of sheep, whilst blood samples were obtained simultaneously from the aorta, the left ventricle, inferior vena cava and the right ventricle; this is a revival of the classical single-injection, multiple-sampling indicator-dilution method. The timing and the area under the indicator dilution curves were calculated. The results showed that at least $80 \%$ of the venous return from the bronchial circulation drained to the left side of the heart, whereas less than $20 \%$ returned to the right heart. In additional studies, Pare also examined the extraction of labelled polymorphonuclear leucocytes (PMNs) injected directly into the pulmonary or the bronchial artery. First-pass extraction of the PMNs was much lower in the bronchial compared to the pulmonary circulation (50 vs 80-90\%), suggesting that some of the anastomoses between the bronchial and the pulmonary vasculature were at the postcapillary level. In summary, results from the dogs and rats demonstrated considerable remodelling of the bronchial circulation and evidence for precapillary anastomoses; whereas, in the acute study in normal sheep, at least some of the anastomoses were postcapillary. At this stage, it does not seem possible to decide whether this is due to species differences or to the effects of angiogenetic remodelling of the bronchial circulation in some studies.

\section{Remodelling of the airway circulation}

Hypertrophy and hyperplasia of the bronchial circulation was evident in cirrhotic rats (D. Schraufnagel), in sheep with lung consolidation (P. Carvalho), in adult dogs 4 months after ligation of the left pulmonary artery (LPA) (R. Michel), and in 8 week old lambs, after ligating the LPA just after birth (N. Charan). In addition, an increased number of dilated bronchial vessels was found in the airway walls of rats infected with mycoplasma, when compared to healthy controls (D. McDonald).

Some insight is being gained into the factors which may be responsible for this angiogenesis. Endothelin-1 (ET-1) may play a role. Its expression in bronchial vessels is increased after ligation of the LPA in dogs, and

*Faculté de Médecine Cochin Port-Royal et Hôpital Cochin, Paris, France. ** Laboratoire de Physiologie, Université de Bordeaux II, Bordeaux, France. +VA Medical Center, Boise, Idaho, USA. +++St Paul's Hospital, Vancouver, Canada. Correspondence: R. Marthan, Laboratoire de Physiologie, Université de Bordeaux II, 146 rue Léo Saignat, 33076 Bordeaux Cedex, France. LIST OF PARTICIPANTS: P. Agostoni (Milan, Italy); L. Baile (Vancouver, Canada); L. Cabanes (Paris, France); P. Carvalho (Boise, USA); N. Charan (Boise, USA); F. Chung (London, UK); M. Corboz (Mobile, USA); V. Doerschuk (Boston, USA); J. Dubrez (Pessac, France); O. Eickelberg (Basel, Switzerland); A. Freed (Baltimore, USA); H. Guénard (Bordeaux, France); M. Hlastala, (Seattle, USA); S. Lakshminaryan (Seattle, USA); C. Le Merre (Nimes, France); A. Lockhart (Paris, France); R. Marthan (Bordeaux, France); R. Matran (Lille, France); D. McDonald (San Francisco, USA); R. Michel (Montréal, Canada); W. Mitzner (Baltimore, USA); N. Neuparth (Lisbon, Portugal); P. Paré (Vancouver, Canada); G. Parsons (Sacramento, CA, USA); D. Pearse (Baltimore, USA); G. Persson (Lund, Sweden); J. Regnard (Besançon, France); A. Rendas (Lisbon, Portugal); P. Robineau (Suresnes, France); J.P. Savineau (Bordeaux, France); D. Schraufnagel (Chicago, USA); J. Souders (Seattle, USA); M. Sparrow (Nedlands, Australia); N. Stephens (Winnipeg, Canada); D. Traber (Galveston, USA); C. Twort (London, UK); P. Vanhoutte (Courbevoie, France); E. Ward (London, UK); A. Wanner (Miami Beach, USA); J. Ward (London, UK); U. Wells (London, UK); S. White (Newcastle, Australia); R. Ziesche (Basel, Switzerland). 
remodelling of the bronchial circulation is reduced by administration of inhibitors of ET-1 in this experimental situation (R. Michel). This is an observation whose relevance to the pathophysiology of the airway circulation is highlighted by the finding that endothelial cells from sheep bronchial arteries express preproendothelin and release ET-1 (A. Wanner). Intravascular pulmonary macrophages may also be involved, since their number is increased in the pulmonary microvasculature of cirrhotic rats (D. Schraufnagel). PMN's are also likely candidates. Numerous hypertrophied vessels were seen to converge towards the site of application of the supernatant of human PMNs in the shell-less chick embryo model. Similar vascular changes of subpleural vessels were also observed through a "window" in the chest wall after local application of interleukin-8 or fibroblast growth factor, suggesting that inflammation may also stimulate angiogenesis (N. Charan).

Remodelling modifies the response of the bronchial vasculature to a variety of stimuli. Firstly, in mycoplasma-infected rats, substance P-induced leakage of monastral blue from the airway vessels is increased, as is the number of neurokinin-1 (NK-1) receptors in the bronchial capillaries; such changes are reversed by treatment with antibiotics or glucocorticoids (D. McDonald). Secondly, when the NO synthase inhibitor $\mathrm{L}-\mathrm{N}^{\mathrm{G}}$-nitroarginine methyl ester (L-NAME), is given to a healthy sheep, the bronchial blood flow decreases by approximately $47 \%$, whereas in sheep with lung consolidation and high baseline bronchial blood flow L-NAME causes an augmented decrease in bronchial blood flow by approximately $73 \%$, suggesting that the control of vascular tone by NO in normal airways is different from abnormal airways (P. Carvalho). Thirdly, although breathing dry air causes no bronchial response in anaesthetized pigs in the control situation, it evokes an early bronchoconstriction in pigs in which previous inhalation of metabisulphite produced marked epithelial sloughing, congestion, oedema and infiltration by PMNs of the airways (R. Matran).

Impressive hypertrophy and hyperplasia of the bronchial vasculature in humans is well-documented in acquired conditions, such as bronchiectasis, lung abcess and lung carcinoma, as well as in congenital heart diseases, with hypoplasia or absence of pulmonary blood flow. Circumstantial evidence suggesting the presence of structural changes of the airway circulation has been obtained in two common clinical conditions; chronic heart failure and asthma. P. Agostoni has observed in patients with longstanding but not recent heart failure, who are undergoing cardiopulmonary bypass, a 5-6 fold increase in blood flow from the aortic root to the left atrium. Although some contribution from the thebesian veins cannot be ruled out, this finding is highly suggestive of hypertrophy and/or hyperplasia of the tracheobronchial circulation in chronic heart failure, and is consistent with the hypothesis that vascular engorgement and/or oedema of the airways make a significant contribution both to nonspecific bronchial hyperresponsiveness and exertional dyspnoea in such patients. In patients who had fully treated left ventricular failure, L. Cabanes found individual differences in the bronchial response to vasoactive agents and physical exercise. She also found that nonspecific bronchial hyperresponsiveness to methacholine, which is probably due to the vasodilator effect of the drug as it is fully prevented by pretreatment with inhaled methoxamine, was present in most but not in all of the patients. Similarly, the improvement in exercise tolerance produced by pretreatment with inhaled methoxamine was more pronounced in some patients than others. J. Regnard reviewed the bronchial effects of inhaled alphaagonist agents and the effects of intravenous infusion of phenylephrine in asthmatic subjects. Alpha-agonist agents are well-documented vasoconstrictors of the tracheobronchial circulation in experimental animals and may cause airway smooth muscle to contract through stimulation of post-junctional alpha-adrenergic receptors. In all human studies, considerable differences were found between asthmatic subjects in their bronchial responses to alphaagonist agents. Indeed, on administration of intravenous phenylephrine the spontaneous bronchial obstruction was aggravated in about half of the subjects and alleviated in the remaining half. Similarly, pretreatment with inhaled methoxamine either reduced, aggravated or did not affect the bronchial response to exercise or inhaled histamine. These findings suggest that the role of vascular congestion and/or oedema was relatively more important than that of bronchospasm in those subjects in whom alphaagonist agents had a favourable effect on the spontaneous or induced bronchial constriction.

In summary, in animals and probably also humans, structural changes of the bronchial circulation, such as remodelling caused by an underlying disease, are capable of modifying vascular and bronchial responses to experimental interventions; a fact that should be borne in mind when interpreting the results of animal or human experiments.

\section{Vasomotor control of the tracheobronchial circulation}

Nerves and ganglia containing a number of cell bodies are present very early in the developing foetal airways of pigs and rabbits. Nerve terminals and evidence of axonal transport, as shown by the presence of synaptin II, are found as far as the most distal airways but do not extend into the epithelial buds. Nerve terminals are found not only within the bundles of airway smooth muscle but also around airway blood vessels. The role of these nerves is not known but the current hypothesis is that they have a trophic role in developing airways (M. Sparrow).

In adult awake sheep, there is distinct evidence in favour of a neural control of the airway vasculature. At rest, the vasomotor tone of the airway circulation results predominently from the balance between alpha-adrenergic and cholinergic tonic influence. Reflex short-lived vasodilatation, elicited by behavioural factors, such as cough, sneezes, sighs and swallowing, and by stimulation of the carotid bodies, are not modified by autonomic blockade and are, in part, dependent on nitric oxide (NO), since the reflex vasodilatation is diminished by the NO synthase inhibitor L-NAME. Stimulation of the distal end of the cut vagi causes a rapid onset vasodilatation not inhibited by cholinergic and adrenergic blockade and by L-NAME. Thus, in addition to the classical autonomic control of tracheobronchial vascular tone, there is presumably an antidromic C-fibre-mediated and a nitroxidergic vasodilatation; whether the NO is of neural or endothelial origin is not known (G. Parsons and S. White). 
In support of a role for neuropeptide-containing $\mathrm{C}$ fibres is the finding that capsaicin causes an increase in the arterial flow of the perfused in situ isolated tracheal segment in the dog (U Wells).

Direct evidence for the vasodilatory effect of inhaled beta $_{2}$-agonists (P. Carvalho) and histamine (L. Baile) on sheep airways, and of the vasoconstrictor effect of phenylephrine in airways of sheep (L. Baile) and dogs (U. Wells) is compelling. However, phenylephrine may have a dual effect on the airway vasculature. This alpha-agonist agent reduces basal bronchial blood flow in anaesthetized sheep pretreated with propranolol and ibuprofen, but reinforces the tracheobronchial vasodilatation caused by hyperosmolar solutions injected into the bronchial artery. When the baseline flow was reduced with phenylephrine to the same extent as with L-nitroarginine there was a greater fractional increase in blood flow following hyperosmolar injections. This suggests that NO mediates part of the hyperosmolar response (L. Baile). The aforementioned results in different animal species are consistent with the finding that alpha-agonist agents vasoconstrict (L. Cabanes, J. Regnard) and histamine and salbutamol vasodilate (H. Guénard) the tracheobronchial circulation in humans.

The role of NO in the control of the tracheobronchial circulation is supported by several pieces of evidence. Firstly, endothelial cells from sheep possess NO-synthase activity, as shown by conversion of L-arginine to L-citrulline (E. Wagner). Secondly, the NO-synthase inhibitor, L-NAME, causes a remarkable decrease in the bronchial blood flow of sheep with consolidated lungs and high baseline bronchial flow and attenuates the isoetharineinduced rise in bronchial blood flow; whereas, inhaled NO (100 ppm) causes an increase in bronchial blood flow even when it is already elevated by pretreatment with isoetharine (P. Carvalho). L-NAME also attenuates the reflex vasodilatory responses to a variety of stimuli in sheep (G. Parsons). Thirdly, L-nitroarginine, a L-arginine analogue, reduces both baseline bronchial blood flow and the bronchial vascular vasodilatory response to ionic and nonionic contrast agents in anaesthetized sheep (E. Baile). The mode of action of NO produced by the endothelium on vascular tone, together with that of other endothelium-derived vasoactive substances was reviewed by P. Vanhoutte. It is noteworthy that several agonists that have been shown to cause vasodilatation of the tracheobronchial circulation, e.g. acetylcholine, are linked to the endothelial-NO pathway. It is tempting to speculate that $\mathrm{NO}$, whose output in the expired gas increases in proportion to minute ventilation on exercise, is an important mediator not only of hyperpnoea- and exerciseinduced tracheobronchial vasodilation, but also of the fall in airway resistance that accompanies physical exercise. The latter assertion is supported by the finding that NO probably exerts a tonic influence to reduce airway resistance in vivo, since pretreatment with L-NAME increases the bronchial response to methacholine in sheep (E. Wagner).

Nitric oxide is not the only paracrine vasomotor mediator in the tracheobronchial circulation. Infusion of microspheres into the bronchial artery causes a dose-dependent vasodilatation. The adenosine receptor antagonist, 8phenyltheophylline attenuated the vasodilatation; whereas, dipyridamole, an inhibitor of adenosine transport, attenuated it. The cyclooxygenase inhibitor indomethacin also reduced the response to microspheres. These results suggest that the microsphere-induced vasodilatation of the bronchial circulation is associated with the release of a vasodilator cyclooxygenase product and of adenosine nucleotides, which are rapidly metabolized to adenosine (D Pearse).

As reviewed by P. Vanhoutte, the endothelial cells produce not only the vasodilator factor NO but also the elusive vasodilator endothelial-derived hyperpolarizing factor (EDHF) and the vasoconstrictor factors endothelin, endoperoxides and thromboxane- $\mathrm{A}_{2}$. The type of mediator produced and the balance of vasoconstrictor and vasodilator mediators depend on the vessels and on the stimulus. Although circumstantial evidence for the release of endothelial-derived vasoactive mediators in the bronchial circulation has been obtained through physiological studies, there is little direct evidence for the actual production of such factors. This is exemplified by the failure to demonstrate the presence of adenosine in the bronchial circulation in spite of the physiological evidence in favour of their release following infusion of microspheres in the bronchial artery (D. Pearse). Similarly, D. Traber was unable to identify mediators in the effluent systemic blood of lungs following cotton smoke-induced acute lung injury in sheep. In this context, it is noteworthy that cultured endothelial cells from sheep bronchial artery have NOsynthase activity (E. Wagner), contain preproendothelin, and release more ET-1 in the culture medium than do bronchial epithelial cells (A. Wanner). In addition, results from careful morphometric studies demonstrated that there are more endothelial than epithelial cells in the airway wall and that the area of endothelial cells in close apposition to airway smooth muscle is far greater than that of epithelial cells (A. Wanner). These observations suggest that the endothelium of the bronchial vessels may play a role in the control of airway smooth muscle, in addition to its role in the control of the vasomotor tone of the bronchial vasculature.

\section{Exchange function of the airway circulation}

The conducting airways of the lungs play an important role in heating and humidifiying of inspired air and in the exchange of highly soluble gases. Gases delivered via the bronchial circulation must cross the capillary walls, the layer of nonperfused tissues and the mucus in order to reach the gas phase in the lumen of the airways. A mathematical model of airway gas exchange has been developed and experiments with an isolated in situ dog trachea preparation and the six inert gases method have been carried out to further our understanding of exchange of gases of varying solubility in the trachea (M. Hlastala and J.E. Souders). The degree of exchange depends critically on the blood:gas partition coefficient, the most soluble gases equilibrating more efficiently. The actual diffusion capacity of the trachea is lower than that estimated from the physical characteristics of the gas. The model predicts that tracheal gas exchange is limited primarily by perfusion- and diffusion-related resistances. This contrasts with the perfusion-limited alveolar gas exchange of $\mathrm{O}_{2}$ and $\mathrm{CO}_{2}$, and is due primarily to the thickness of the bronchial mucosa. These considerations apply to the tracheal exchange of alcohol. When the ratio 
of partial pressure of alcohol in the expired gas to that in the alveolar gas is plotted as a function of the exhaled volume, there is a minimal dead space for alcohol. Alcohol in the bronchial blood equilibrates with the inspired air in the conducting airways and not within the alveoli. On expiration, some $20-50 \%$ of the alcohol released from the airways during inspiration is redeposited to the airways, depending on the breathing manoeuvre. Water vapour is also exchanged in the conducting airways, such that the inspired gas is saturated on inspiration and a significant fraction of the water is redeposited on expiration. The efficiency of water conservation is rather low in humans compared to some desert mammals, in whom expired gas at the nostrils is likely to be completely dry (H. Guénard).

The airway circulation plays a major role in clearance of exogenous material deposited on the airway. Absorption of low molecular weight tracers from the tracheal lumen to the bronchial vessels has been studied in the isolated, perfused in situ trachea of dogs (U. Wells). U. Wells measured the venous output and the permeability coefficient of the lipophilic ${ }^{14} \mathrm{C}$-antipyrine and of the hydrophillic $99 \mathrm{~m}$ Tc-diethylenetriamine pentaacetic acid (DTPA) with molecular weight of $188 \mathrm{kDa}$ and $492 \mathrm{kDa}$, respectively. The permeability coefficient of DTPA was much lower than that of antipyrine and increased when the airway epithelium was injured by local application of either a detergent or hydrogen peroxide. Based upon these results, she suggested a paracellular pathway for the passage of DTPA across the airway epithelium along the diffusion gradient. However, one cannot rule out active transport of DTPA across the airway epithelium superimposed upon the paracellular passage. Indeed, there is mounting evidence that hydrophilic organic anions are actively transported across the airway epithelium and that the transport mechanism shares many properties with that of other epithelia.

Although U. Wells found an inverse relationship between bronchial blood flow and lumen-to-vessel transport of DTPA, others found opposite results. In anaesthetized sheep, the reduction of bronchial blood flow delayed the recovery of methacholine-induced bronchoconstriction, an effect which was best explained by reduced wash-out of the drug from the airways. In addition, the retention of ${ }^{99} \mathrm{mTc}$-sulphur colloid particles with a $2 \mu \mathrm{m}$ diameter deposited in central airways was greatly prolonged when bronchial blood flow was interrupted (E. Wagner). These experiments in animals suggest that changes in tracheobronchial blood flow are probably important determinants of the bronchial response to most, if not all, bronchial provocation tests in human studies. And one also wonders whether changes in airway blood flow might play a role in the clearance of endogenous mediators released in the airway wall in clinical situations characterized by damage to the airways (D. Traber), although, as already mentioned, there is as yet no direct confirmatory evidence in favour of this hypothesis.

\section{Plasma extravasation}

C. Persson suggested that plasma extravasation may be a defence mechanism to neutralize noxious substances penetrating the lamina propria and to rinse the luminal surface of the airways. In addition, when the epithelium is abraded, extravasation leads to the formation of a plasma gel covering the denuded basement membrane. Experiments in guinea-pigs have shown that epithelial repair begins minutes after abrasion of the tracheal epithelium by migration from the edges of the wound of dedifferentiated epithelial cells. Permeability of the newly formed epithelium is low, probably because the number of intercellular junctions is reduced due to the larger size of the dedifferentiated cells. This experimental finding is consistent with the reduced nasal absorption of ${ }^{51} \mathrm{Cr}-$ ethylenediamine tetraacetic acid (EDTA) during the pollen season in subjects who have allergic rhinitis. The defence role of plasma extravasation was also outlined by A. Freed, who found, in experiments in dogs, that bronchovascular vasodilation and leakage protect the airway mucosa of distal airways from injury induced by hyperventilation of dry air.

Plasma extravasation associated with epithelial shedding (C. Persson), dry air ventilation in pigs pretreated with metabisulphite (R. Matran), and cotton smokeinduced acute lung injury (D. Traber), is associated with margination and extravascular migration of PMNs in the airway wall. The molecules mediating PMN adhesion in the bronchial circulation are just beginning to be identified. Results from recent studies, in which anti-CD11a and CD11b and anti-platelet-endothelial-cell-adhesion molecule 1 (PECAM 1) antibodies were used suggest the existence of CD11/CD1- and PECAM-1-independent pathways for adhesion of PMNs in the bronchial circulation. It is noteworthy that emigration of PMNs from the bronchial and from the pulmonary circulation is similar but differs considerably from other systemic vascular beds, suggesting that adhesive interactions and transendothelial migration may occur through mechanisms that are regulated primarily by the environment within the lung and by which cytokine production by resi-dent cells is induced by the stimulus used (C. Doerschuk). Attracted PMNs release oxygen radicals and proteases resulting in injury to the airways (D. Traber).

The site of extravasation has been studied in airways of rats by D. McDonald. Extravasation, as assessed by use of the monastral blue dye, evoked by bradykinin, histamine and substance $\mathrm{P}$, took place in postcapillary venules up to $80 \mu \mathrm{m}$ in diameter. It was associated with the formation of gaps between endothelial cells. The gaps were less than $1 \mu \mathrm{m}$ in diameter and occupied less than $3 \%$ of the luminal surface of the venules. They appeared to be formed by localized retractions of the endothelial plasma membrane rather than by generalized contraction of the endothelial cells. The gaps occurred within minutes after application of substance $\mathrm{P}$ and had a half-life of a few minutes. Their localization matched that of tachykinin receptors of the NK-1 subtype. The rapid closure of the substance P-induced pores was due to rapid internalization of the receptors. In mycoplasmainfected rats, both the transendothelial leakage and number of NK-1 receptors were increased. Conversely, pretreatment of rats with a long-acting beta ${ }_{2}$-agonist reduced by the same amount, the leakage and the number of NK-1 receptors. However, in dogs, dry air hyperventilation caused a long-lasting extravasation in the airways (A. Freed) and its duration suggests another mechanism of transendothelial passage than that described in rats (D. McDonald). 
The effects of extravasation on the wall thickness of airway vessels has been investigated by E. Wagner and W. Mitzner. E. Wagner showed that the airway wall area of airways of $1 \mathrm{~mm}$ in diameter increased up to 30-40\% after infusion of bradykinin into the bronchial artery of sheep. The increase in wall thickness took place external to the smooth muscle layer and did not result in airway obstruction. W. Mitzner used high-resolution computed tomography (CT) to study the thickness of the airway wall in dogs in airways ranging from $2.1-2.6 \mathrm{~mm}$ in internal diameter. Infusion of blood $\left(150 \mathrm{~mL} \cdot \mathrm{kg}^{-1}\right)$ resulted in dilation of the vessels with almost no change in the thickness of the airway walls, and caused some reduction of the lumen of the airways. Infusion of saline (50 $\left.\mathrm{mL} \cdot \mathrm{kg}^{-1}\right)$ caused an increase in airway wall thickness, a decrease in luminal area, and almost no change in the external diameter of the airways. The maximal changes in wall thickness and lumen area were +50 and $-35 \%$, respectively. Similarly, infusion of bradykinin in dogs pretreated with atropine, caused an increase in wall thickness and a decrease in the lumen of the airways. The maximal increase in wall thickness seems to be insufficient by itself to cause a major degree of airway obstruction but enhanced the bronchial response to histamine.

In summary, vascular leakage in the airway wall may be a double-edged sword. It may facilitate clearance of extravasated substances and reduce acute lung injury in some experimental solutions. But it may also be associated with transendothelial migration and activation of PMNs leading to tissue injury and to worsening of airway obstruction and constriction in other experimental situations. The respective protective and deleterious roles of vascular congestion and leakage in human diseases remain poorly understood.

\section{Smooth muscle in the lung}

Since physiological and pathophysiological responses of the bronchial circulation ultimately depend on the functioning of a particular type of cell, i.e. the smooth muscle cell which is also an important component of airway and pulmonary artery walls, a session of the symposium was devoted to the cellular pathophysiology of "pulmonary" smooth muscles. Recent issues regarding some of the cellular mechanisms involved in smooth muscle contraction were discussed in the context of airway and/or pulmonary smooth muscle. Using a preparation of isolated human bronchial smooth muscle cells chemically skinned by addition of beta-escin (i.e. muscle fibres in which cell membranes are made permeable to ions), the concept of alterations in sensitivity to calcium of the contractile apparatus was illustrated (R. Marthan). In particular, it was shown that cyclic adenosine monophosphate (cAMP) and cyclic guanosine monophosphate (cGMP) both decrease this sensitivity, although by mechanisms that may not be similar. Direct measurements of the variation of the cytosolic concentration of free calcium ions $\left[\mathrm{Ca}^{2+} \mathrm{i}\right]$ in isolated pulmonary smooth muscle cells activated by agonists have been made by means of fluorescent dyes, such as indo 1 or fura 2 . In airways, the increase in $\left[\mathrm{Ca}^{2+\mathrm{i}}\right]$ is graded, i.e. there is a concentration-response relationship between the concentration of the agonist and the $\left[\mathrm{Ca}^{2+i}\right]$ value which is similar to that of the development of isometric force ( $R$. Marthan). In contrast, in the pulmonary artery, the $\left[\mathrm{Ca}^{2+} \mathrm{i}\right]$ response to angiotensin II is an all or none phenomenon (J.P. Savineau). The use of microspectrofluorometry with calcium-sensitive dyes has provided new insight into the study of $\mathrm{Ca}^{2+}$ accumulating and releasing functions of intracellular calcium stores in smooth muscle. In airways, C. Twort demonstrated that the sarco-plasmic reticulum is the main store of calcium in smooth muscle and that it acts both as a source and a sink for the control of $\left[\mathrm{Ca}^{2+\mathrm{i}}\right]$. Moreover, when located close to the cell membrane, it also acts as a superficial buffer barrier. In the pulmonary artery, J.P. Savineau showed that angiotension II produced intracellular calcium oscillations due to the cycling of $\mathrm{Ca}^{2+}$ from and to the sarcoplasmic reticulum. These oscillations are related to a complex control of the inositol $(1,4,5)$ triphosphate $\left(\mathrm{IP}_{3}\right)$ receptor-channel protein, since they do not occur when the ryanodine calcium release channel is stimulated, as for example by caffeine. As in the airway smooth muscle, the calcium sensitivity of the contractile apparatus can be modulated in the pulmonary vascular muscle. For example, activators of protein kinase $\mathrm{C}$, such as phorbol esters, increase the calcium sensitivity of the contractile apparatus to such an extent that they induce "calcium-independent" contraction, i.e. the contraction occurs in the absence of detectable increase in $\left[\mathrm{Ca}^{2+} \mathrm{i}\right]$. J. Ward reviewed some electrophysiological properties of pulmonary smooth muscles and pointed out specific features regarding potassium conductance in these muscles. In pulmonary arterial smooth muscle cells, hypoxic vasoconstriction appears to be due to a decrease in the outward current flowing through the delayed rectifier $\mathrm{K}^{+}$channel; whereas, in human airway smooth muscle, much of the outward $\mathrm{K}^{+}$ current flows through $\mathrm{Ca}$-activated $\mathrm{K}^{+}$channels $\left(\mathrm{BK}_{\mathrm{Ca}}\right.$ or maxi-K or charybdotoxin-sensitive $\mathrm{K}$ channel). This specificity may be clinically relevant, since the functioning of the $\mathrm{BK}_{\mathrm{Ca}}$ can be modulated by pharmacological compounds that can be given to humans. Finally, N. Stephens presented data regarding phenotypic modulation of pulmonary smooth muscle cells associated with remodelling as occurs, for example, in chronic asthma. In the course of cell culture, changes in the phenotype of airway smooth muscle cells is accompanied by changes in protein profiles. Cultured dedifferentiated "noncontractile" cells expressed less tropomyosin, myosin light chain kinase than freshly dispersed differentiated cells but more non-muscle myosin, simentin and 1-caldesmon. Similar differences in protein profiles are observed between slow smooth muscles, such as the pulmonary artery, and fast ones, such as the trachealis. It is likely that these changes in protein profiles also occur in vivo.

\section{Conclusion}

Until a few years ago, the physiological role of the collateral lung circulation was not fully appreciated. Indeed, the size and site of anastomoses between the bronchial and pulmonary circulation, the magnitude and direction of the ensuing shunt and the role of the bronchial circulation in pulmonary gas exchange in congenital heart diseases with a right-to-left shunt, were the object of many studies some decades ago. More recently, the 
advent of lung transplantation has caused a renewed interest in the nutritional role of the airway circulation and has led to the realization that the site of the anastomosis of the recipient and donor lung was important to ensure proper vascularization and healing of the suture line. The preliminary observation of J. Dubrez that bronchial artery revascularization leads to a reduced incidence of obliterative bronchiolitis in lung-transplant recipients, although as yet unconfirmed, opens new horizons with respect to the role of the airway circulation in the maintenance of the normal structure of the airway. Although oedema and vascular congestion of the airway wall in conditions such as asthma and chronic heart failure have been known for many decades, it is only in recent years that a systematic study was undertaken of the mechanisms and consequences to the airway physio-logy of congestion and leakage in the airway circulation. During this conference, it became apparent that the response of the airway circulation to a variety of stimuli is greatly affected by the prevailing environment in the airway wall. Examples of the influence of inflammation and remodelling on the vascular and bronchial responses to a variety of stimuli have been highlighted during the meeting and should be borne in mind when undertaking future studies.

Acknowledgements: The symposium was sponsored by INSERM and the European Respiratory Society. The organizers of the Symposium are indebted to the following sponsors: INSERM, Conseil Régional d'Aquitaine, Astra-Draco International, Glaxo (USA), Laboratoires Fisons (France), Institut de Recherches Servier (France), Pfizer (USA), and Synthélabo Pharmacie (France). 